\title{
Helical spring design optimization for endoscopic devices using a design-of- experiments and response surface approach
}

\author{
Hamid Ebrahimi, PhD \\ Holland Musculoskeletal Research Program, \\ Sunnybrook Research Institute \\ IBBME, University of Toronto \\ Toronto, Canada \\ hamid.ebrahimi@mail.utoronto.ca \\ Albert Yee, MSc, MD, FRCSC \\ Holland Musculoskeletal Research Program, \\ Sunnybrook Research Institute \\ Dept of Surgery and IBBME, University of Toronto \\ Toronto, Canada \\ albert.yee@sunnybrook.ca
}

\author{
Cari Whyne, $\mathrm{PhD}$ \\ Holland Musculoskeletal Research Program, \\ Sunnybrook Research Institute \\ Dept of Surgery and IBBME, University of Toronto \\ Toronto, Canada \\ cari.whyne@sunnybrook.ca
}

\begin{abstract}
Flexible endoscopes require reliable advancement and steerability of the device tip. Helical spring design is critical to both endoscopic steerability and cable function. To characterize the impact of geometric and material factors on endoscopic function, a parametric helical spring and a cable assembly was modelled using the finite element method and analyzed using a design-of-experiments approach. Individual input parameters (height, modulus (E), force, and width) and two interactions (pitch/width and pitch/height/E/width) were found to significantly impact the radius of curvature (a measure of steerability). The force and pitch/width interaction had negative effects, in contrast to positive effects from height, E, width and the pitch/height/E/width interaction. This information provides critical geometric and material information to guide helical spring design for optimized endoscopic steerability.
\end{abstract}

Keywords: Endoscope, Helical Spring, Finite Element Analysis, Design-of-Experiments, Geometry and Material Parameters, Radius of Curvature

\section{INTRODUCTION}

Endoscopes are long thin tubular devices for non-invasive visualization of the interior of cavities, canals and vessels. The flexibility of articulated endoscopes is important in many clinical applications. Articulated endoscopes are generally controlled by cables and levers which bend the distal extremity of the device to enable route selection or to change the field of view.

Challenges associated with flexible endoscope design include reliable advancement and steerability of the device tip [1]. Reliable advancement can be addressed by utilization of relatively rigid helical springs within the device shaft. The helical geometry provides bending flexibility at the expense of some reduction in axial stiffness and tensile strength [2]. However, the device tip must be flexible for steerability to accommodate different bend radii. The device tip can be steered via a cable attached to a control mechanism within the endoscopic handle.

In order to achieve tight bends while preventing cable malfunctions [3] a thorough understanding of the helical spring stress distribution under specific load conditions is necessary. Multiple studies have developed analytical and numerical models to characterize stress distribution in helical springs under axial and pure bending loads [4]. However, to date studies have not considered the optimization of bend radii representative of helical spring and cable assemblies in traditional endoscopes. As such, the aim of this study is to understand the impact of endoscopic tip design (geometry and material properties) on the radius of curvature governing steerability in traditional endoscopes.

\section{METHODS}

\section{A. Experimental Design}

A two-level five-factor design-of-experiments methodology was utilized to understand the main geometric and material effects and their interactions on the attainable radius of curvature of an endoscopic device (outcome). The effects of width, height, pitch, Young's modulus (E) and applied force were assessed at combination of high and low factor levels, resulting in 32 experimental groups (Table 1). The range for width, height and pitch were based on commercially available endoscopic designs, with $\mathrm{E}$ representing metals from Aluminum ( 70GPa) to Steel ( 200GPa). Force ranged from $0.5 \mathrm{~N}$ (to allow the device tip in the most rigid configuration to 
displace at least $0.1 \mathrm{~mm}$ ) to $2 \mathrm{~N}$ (representing the maximum tensile force a $0.1 \mathrm{~mm}$ diameter steel cable can withstand before plastic deformation).

\begin{tabular}{|l|l|l|l|l|l|}
\hline $\begin{array}{c}\text { Factor } \\
\text { Level }\end{array}$ & $\begin{array}{c}\text { Width } \\
(\mathbf{m m})\end{array}$ & $\begin{array}{c}\text { Height } \\
(\mathbf{m m})\end{array}$ & $\begin{array}{c}\text { Pitch } \\
(\mathbf{m m})\end{array}$ & $\begin{array}{c}\text { E } \\
(\mathbf{G P a})\end{array}$ & $\begin{array}{c}\text { Force } \\
(\mathbf{N})\end{array}$ \\
\hline Low & 0.1 & 1 & 3 & 70 & 0.5 \\
\hline High & 1 & 2.5 & 4 & 200 & 2 \\
\hline
\end{tabular}

TABLE I. HIGH AND LOW FACTOR LEVELS ARE PRESENTED FOR WIDTH, HEIGHT, PITCH, E AND FORCE USED TO INVESTIGATE THE ENDOSCOPIC GEOMETRIC AND MATERIAL EFFECTS AND THEIR INTERACTIONS ON THE ACHIEVABLE RADIUS OF CURVATURE

\section{B. Finite Element (FE) Model Generation}

An FE model of a traditional endoscope was generated that enabled parametric geometric and material property assignments (Table 1). The helical spring (rectangular cross section) was modelled using linear brick elements. The cable was modelled using linear 3-D truss elements. Once assembled the cable proximal end was connected to the helical spring proximal outer circumference (Fig. 1a) and the cable distal end was placed inside the helical spring (Fig. 1b). Interactions were modelled using a general contact algorithm. An ENCASTRE boundary condition was used to fix the distal end of the helical spring. The cable was connected to the helical spring tip using a tie constraint. A concentrated force was then applied to the cable distal end to simulate the pulling force from the controller unit and analysed in ABAQUS dynamic/explicit.

The radius of curvature was calculated based on spatial displacement outputs identified between a node defined at the cable attachment site on the helical spring tip and the most distal node with greater than zero displacement at the base of the helical spring. Mesh convergence analysis was performed by refining the mesh size and calculating the radius of curvature and maximum deformation in each iteration (to within 5\%). The final model contained 3692 nodes, 2135 elements and 10920 degrees of freedom.

An automated procedure through the ABAQUS Scripting Interface (ASI) was utilized to create parts, assign material properties, assemble parts, apply boundary conditions and force, and solve each generated model. The generated nodal displacement outputs and deformed geometry image data sets were analysed by a python script to calculate the radius of curvature, annotate images with their corresponding configuration parameters and organize the raw data to be used for design-of-experiments analysis (Fig. 1).

\section{Design-Of-Experiments}

Thirty-two configurations were analyzed in the two-level factorial design-of-experiments analysis (Design-Ease). Based on the Box-Cox plot, a base 10 Log transformation of the output response (radius of curvature) was used to stabilize the variance. A Sum-of-Squares chart with a $2 \%$ weighted contribution threshold was utilized to determine the model inclusion criteria. A Shapiro-Wilk test was also conducted to confirm data normality. An Analysis of Variance (ANOVA) with a Bonferroni correction was conducted to determine the significance of the model, effects and interactions.

\section{RESULTS}

All thirty-two models were solved successfully. The model, four of the input parameters (width, height, E, force) and two interactions (between pitch/width and pitch/height/E/width)
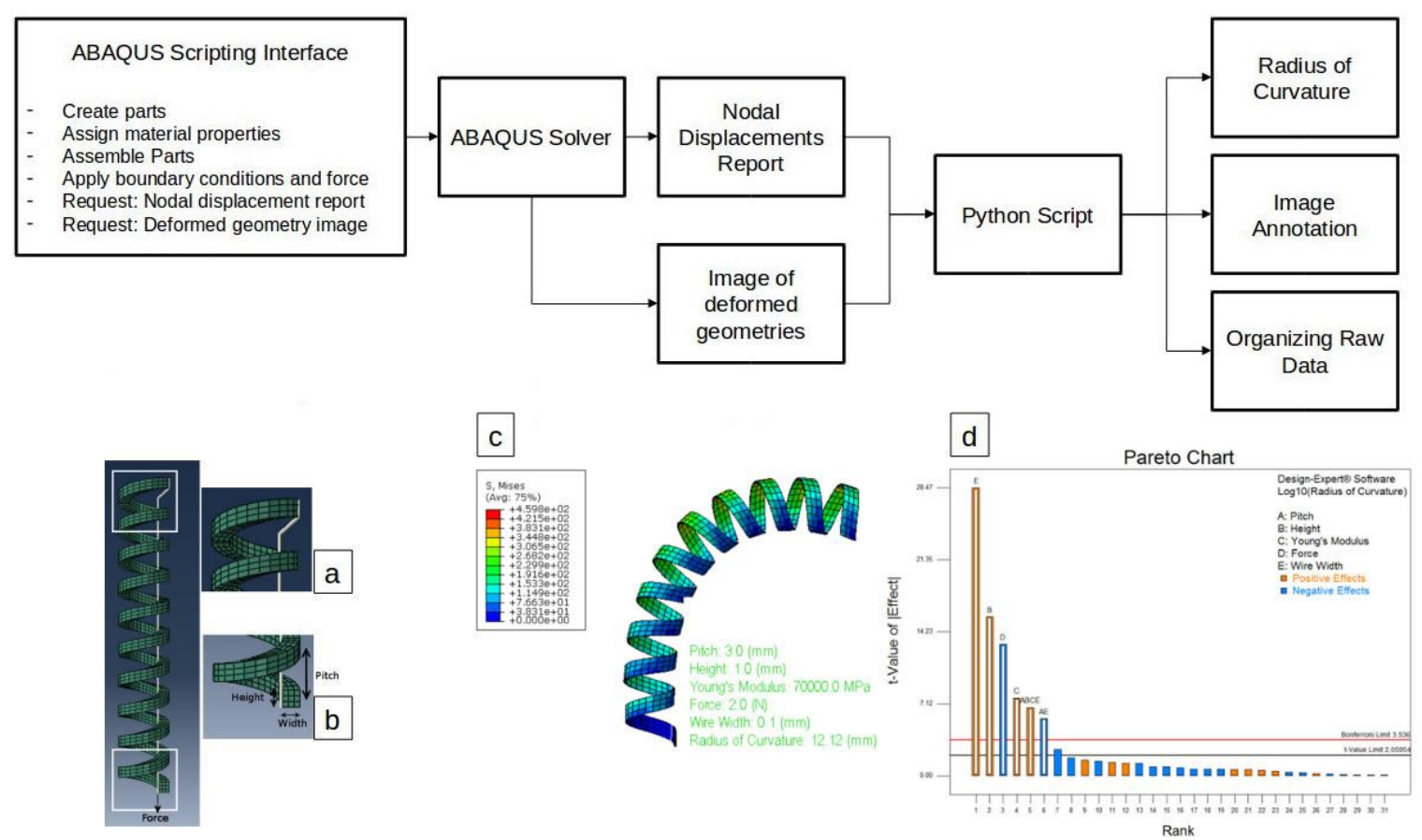

Figure 1. A flowchart describing the automation of the FE model creation and DOE analysis. The unloaded spring model and its parameters are shown (left, $a$ and $b$ ) with the lowest radius of curvature model presented (centre, c). The Pareto chart (right, d) identifies the significant effects and interactions. 
were found to be statistically significant $(\mathrm{P}<0.0001)$. The lowest radius of curvature achieved was $12.12 \mathrm{~mm}$ (Fig. 1c). A Pareto chart summarizing the main effects and model errors is shown in Fig. 1d.

The largest contribution to the model was the width of the spring (effect=1.39, 58.33\% contribution). The force and pitch/width interaction each had a negative influence, whereas the height, E, width and pitch/height/E/width interaction showed a positive influence. A negative effect in which higher forces reduce the radius of curvature allows for tighter bends. The negative AE effect shows that pitch and width work in opposition with respect to their impact on radius of curvature, as evident in the response shown in Fig. 2. Pitch and all other interaction effects below the $2 \%$ contribution threshold were applied as model error. A summary of the statistical data as well as the final equation in terms of coded factors are presented in Table 2 .

\section{DISCUSSION AND CONCLUSION}

A robust computational model was developed using a design-of-experiments approach that allows parametric optimization of endoscopic tip design parameters for radius of curvature minimization. Based on this model some of the identified disadvantages of traditional endoscopes can be addressed by altering design parameters for different applications. A small pitch and width yield a radius of curvature optimal for acute bending radii. If due to design restrictions a larger width is necessary, an increase in pitch may employed to maintain a sufficiently small bending radius.

The challenges associated with endoscopic device advancement have been reported in multiple studies [5,6,7]. A study of FDA reports from 1985 to 2009 (Chen et al. 2010) reported that $21 \%$ of endoscopic mechanism failures are due to cable malfunctions. Based on our models, if advancement of
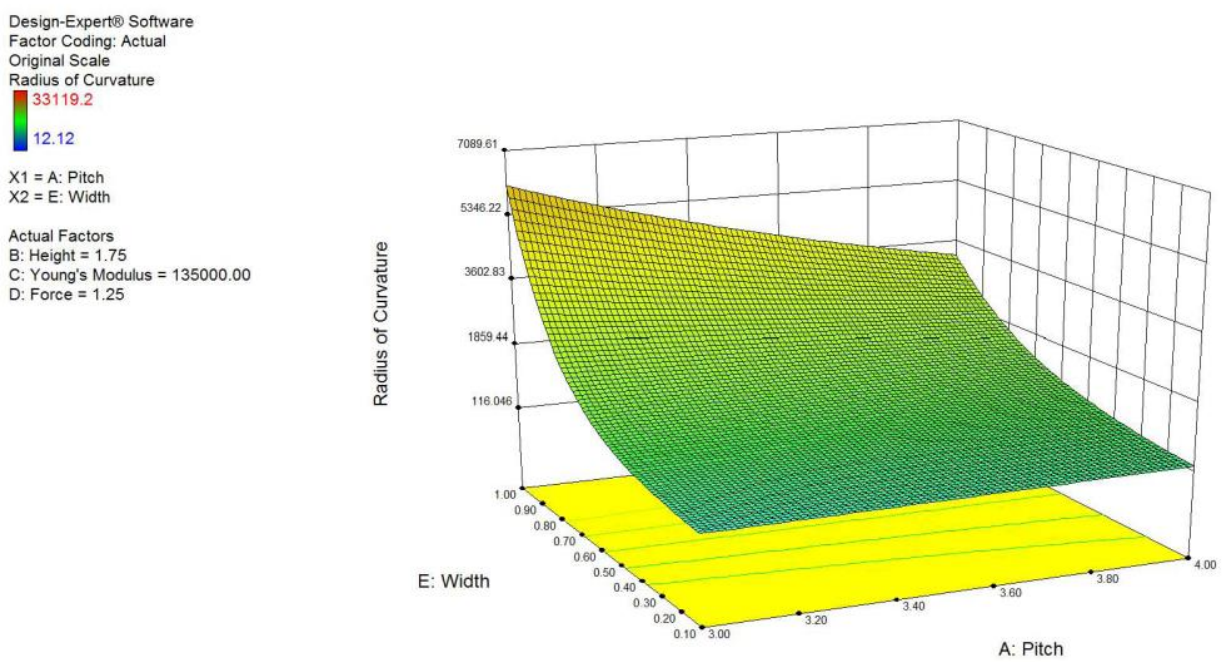

Figure 2. A response surface describing the negative interaction between helical spring pitch and width with respect to radius of curvature.

\begin{tabular}{|c|c|c|c|c|c|c|}
\hline $\begin{array}{c}\text { Applied } \\
\text { Transformation }\end{array}$ & Source & $\begin{array}{l}\text { Coded } \\
\text { Factors }\end{array}$ & Effect & Contribution (\%) & F Value & P Value \\
\hline \multirow{7}{*}{$\begin{array}{l}\text { Base } 10 \\
\text { Logarithm }\end{array}$} & Model & NA & NA & NA & 227.41 & $<0.0001$ \\
\hline & Width & $\mathrm{E}$ & 1.39 & 58.33 & 810.45 & $<0.0001$ \\
\hline & Height & $\mathrm{B}$ & 0.77 & 17.82 & 247.54 & $<0.0001$ \\
\hline & Force & $\mathrm{D}$ & -0.63 & 12.19 & 169.30 & $<0.0001$ \\
\hline & Young's Modulus (E) & $\mathrm{C}$ & 0.37 & 4.26 & 59.23 & $<0.0001$ \\
\hline & Pitch/Height/E/Width & $\mathrm{ABCE}$ & 0.33 & 3.29 & 45.72 & $<0.0001$ \\
\hline & Pitch/Width & $\mathrm{AE}$ & -0.28 & 2.32 & 32.20 & $<0.0001$ \\
\hline Final Equation & \multicolumn{6}{|c|}{$\log ($ radius of Curvature $)=2.96+0.38 * \mathrm{~B}+0.19 * \mathrm{C}-0.32 * \mathrm{D}+0.69 * \mathrm{E}-0.14 * \mathrm{~A} * \mathrm{E}+0.16 * \mathrm{~A} * \mathrm{~B} * \mathrm{C} * \mathrm{E}$} \\
\hline
\end{tabular}

TABLE II. 
the device requires high pushing forces, the device axial stiffness must be increased by increasing the cable width and /or modulus of elasticity and the acute bend radius can still be achieved by increasing the pitch, and/or lowering the height without increasing the force applied to the cables.

This study demonstrates the potential of a combined FE modelling DOE approach for optimization in biomedical instrumentation design. Through optimization of geometric and material properties, endoscopes can be optimized to meet specific tip design criteria and function safely and effectively within varied environmental conditions.

\section{ACKNOWLEDGMENT}

This work was supported by a CIHR Banting and Best scholarship.

\section{REFERENCES}

[1] Menciassi A, Park JH, Lee S, et al. 2002. Robotic solutions and mechanisms for a semi-autonomous endoscope. IEEE/RSJ Int Conf Intell Robot Syst. 2:1379-1384.

[2] Raoof M, Davies TJ. 2004. Determination of the bending stiffness for a spiral strand. J Strain Anal Eng Des. 39:1-13.

[3] Chen Y, Tanaka S, Hunter IW. 2010. Disposable endoscope tip actuation design and robotic platform. Annu Int Conf IEEE Eng Med Biol Soc EMBC'10.:2279-2282.

[4] Keller SG, Gordon AP. 2011. Equivalent stress and strain distribution in helical compression springs subjected to bending. J Strain Anal Eng Des. 46:405-415.

[5] Rex DK, Khashab M, Raju GS, et al. 2005. Insertability and safety of a shape-locking device for colonoscopy. Am J Gastroenterol. 100:817820.

[6] Loeve AJ, Plettenburg DH, Breedveld P, et al. 2012. Endoscope shaftrigidity control mechanism: "FORGUIDE". IEEE Trans Biomed Eng. 59:542-51.

[7] Adebar T, Greer J, Laeseke P, et al. 2015. Methods for Improving the Curvature of Steerable Needles in Biological Tissue. IEEE Trans Biomed Eng. 9294:1-1. 